\title{
NextGen Brain Microdialysis: Applying Modern Metabolomics Technology to the Analysis of Extracellular Fluid in the Central Nervous System
}

\author{
Chi-Ya Kao a,c Elmira Anderzhanova ${ }^{b}$ John M. Asarad, e Carsten T. Wotjak ${ }^{b}$ \\ Christoph W. Turck ${ }^{a}$
}

Departments of ${ }^{\mathrm{a}}$ Translational Research in Psychiatry and ${ }^{\mathrm{b}}$ Stress Neurobiology and Neurogenetics, Max Planck Institute of Psychiatry, and ' Graduate School of Systemic Neurosciences, Ludwig Maximilian University, Munich,

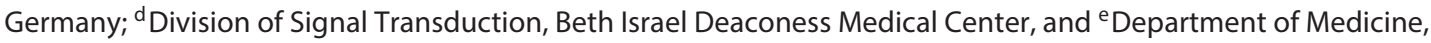
Harvard Medical School, Boston, Mass., USA

\section{Key Words}

Microdialysis · Metabolite profiling · Posttraumatic stress disorder · Molecular pathways · Biomarker · Stress susceptibility and resilience

\begin{abstract}
Microdialysis is a powerful method for in vivo neurochemical analyses. It allows fluid sampling in a dynamic manner in specific brain regions over an extended period of time. A particular focus has been the neurochemical analysis of extracellular fluids to explore central nervous system functions. Brain microdialysis recovers neurotransmitters, low-molecular-weight neuromodulators and neuropeptides of special interest when studying behavior and drug effects. Other small molecules, such as central metabolites, are typically not assessed despite their potential to yield important information related to brain metabolism and activity in selected brain regions. We have implemented a liquid chromatography online mass spectrometry metabolomics platform for an expanded analysis of mouse brain microdialysates. The method is sensitive and delivers information for a far greater number of analytes than commonly used electrochemical and fluorescent detection or biochemical assays. The metab-
\end{abstract}

olomics platform was applied to the analysis of microdialysates in a foot shock-induced mouse model of posttraumatic stress disorder (PTSD). The rich metabolite data information was then used to delineate affected prefrontal molecular pathways that reflect individual susceptibility for developing PTSD-like symptoms. We demonstrate that hypothesis-free metabolomics can be adapted to the analysis of microdialysates for the discovery of small molecules with functional significance.

(c) 2015 S. Karger AG, Basel

\section{Introduction}

Microdialysis is used to dynamically monitor endogenous and exogenous substances in vivo. The procedure can be performed in any tissue [1] and body fluid [2,3]. A major focus is the analysis of brain neurotransmission, neurochemistry and metabolism $[4,5]$, and microdialysis remains the method of choice for experimental neuropharmacology studies by acquiring pharmacokinetic and pharmacodynamic data from living animals [6]. For this purpose, extracellular fluids from selected brain areas are analyzed for molecular components relevant for the 
physiological/pathological processes or drug response. The technique enables an assessment of the release and metabolism of neuroactive endogenous substances over a period of several days in freely moving animals. Sampling of specific brain regions, such as the medial prefrontal cortex (mPFC), paraventricular nucleus and amygdala, during behavioral experiments with rodents can be done with efficient spatial and temporal resolution of millimeters and minutes, respectively, reflecting the actual state of the animal under investigation.

Perfusion of an implanted microdialysis probe with artificial cerebrospinal fluid that resembles the brain extracellular fluid with regard to $\mathrm{pH}$, ion composition and osmolarity is not accompanied by significant fluid gain or loss and tissue homeostasis disturbances, thus making microdialysis a truly physiological method for the purpose of in vivo sampling. By using a semipermeable membrane designed for microdialysis probes, macromolecules such as enzymes and proteins are excluded. This leaves collected extracellular matrices intact. Furthermore, the absence of macromolecular complexes in microdialysates does not require sample purification prior to analysis [5].

Commonly employed methods for the analysis of microdialysates include electrochemical, UV and fluorescence detection coupled with high-performance liquid chromatography (HPLC) as well as radioimmunoassay, enzyme-linked immunosorbent assay, colorimetric and spectrophotometric biochemical assays $[1,7]$.

The analytical methods currently used have restricted more comprehensive in-depth studies of the precious microdialysate samples. Small sample volumes limit the number of molecules, which can be simultaneously analyzed with good sensitivity using the classical detection methods. For this reason, microdialysate collection times are frequently extended, resulting in poor temporal resolution. Modern analytical methods need to be implemented in order to fully exploit microdialysis sample contents. Liquid chromatography online mass spectrometry (LC-MS) allows selective identification and sensitive quantification of a great number of molecules. Although LC-MS methods have been developed for the identification and quantification in microdialysates of neurotransmitters [8-10], neuropeptides [11] and exogenous compounds such as drugs and their metabolites [12-14], most studies have been restricted to a small number of analytes and consequently have resulted in only narrow insights in pathophysiological mechanisms.

LC-MS has become an important method in the field of metabolomics, the comprehensive analysis of metabo- lites. When configured in the selected reaction monitoring (SRM) mode, a great number of molecules can be identified with high confidence. This makes LC-MS the method of choice as a comprehensive screening tool for microdialysate analysis and pathway discovery pertinent to pathophysiological responses.

In the present study, we have profiled and monitored central metabolite levels in microdialysates on a large scale for the first time, using an LC-MS metabolomics platform. We have applied this approach to a posttraumatic stress disorder (PTSD) mouse model $[15,16]$ and have correlated metabolite levels measured prior to foot shock (FS) stress with behavioral readouts of FS resilience (early and delayed post-shock changes in arousal levels). The method is orders of magnitude more comprehensive in terms of qualitative and quantitative molecular information than traditional microdialysate analysis. This information can be exploited to gain a better understanding of pathophysiological mechanisms in mouse models.

\section{Materials and Methods}

\section{Animals}

Male C57BL/6NCrl mice, 7-8 weeks old, (Charles River GmbH, Sulzfeld, Germany) were housed in groups of four in Makrolon type II cages $\left(23 \times 16.5 \times 14 \mathrm{~cm}^{3}\right)$ under an inverse $12: 12 \mathrm{~h}$ lightdark cycle (light off at 7:00) with food and water ad libitum. The experimental procedures were approved by the Government of Upper Bavaria. Laboratory animal care and the experiments were performed in compliance with the European Economic Community recommendations for the care and use of laboratory animals (2010/63/EU).

\section{Fear Conditioning and Acoustic Startle Response}

Fear conditioning for the PTSD mouse model was performed as described previously [15]. In brief, after $198 \mathrm{~s}$ of habituation, the mice perceived two electric FS $(1.5 \mathrm{~mA}, 2 \mathrm{~s})$ with a 60 -second interval in between at moderate illumination (40 Lux) in a plexiglass shock chamber. The mice remained in the shock chamber for another $60 \mathrm{~s}$ before they were returned to their home cages. To test for arousal levels, acoustic startle response (ASR) was measured automatically as described before [16]. The level of ASR was assessed on days 1 and 2 (ASR1 and ASR2) and FS-stimulated ASR changes (ASR2/ASR1, \%) were calculated. For the evaluation of PTSD-like symptoms, the levels of ASR and contextual fear response (CF) were assessed on day 34 (ASR34 and CF34).

\section{Brain Microdialysis}

Guide cannulas were implanted into the right mPFC (coordinates: AP: $+2.20 \mathrm{~mm}$, ML: $0.35 \mathrm{~mm}$ and DV: $-1.50 \mathrm{~mm}$ ) in accordance to Paxinos and Franklin's Mouse Brain Atlas [17] under isoflurane (Abbot, Mumbai, India)/Metacam ${ }^{\circledR}$ (Boehringer Ingelheim $\mathrm{GmbH}$, Ingelheim am Rhein, Germany) anesthesia/anal- 


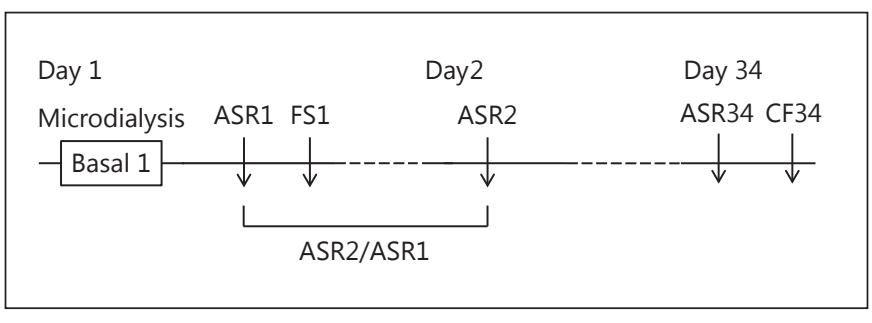

Fig. 1. Outline of the microdialysis experiment in combination with metabolomics applied to a PTSD mouse model. On experimental day 1, ASR1 was measured, followed by fear-conditioning FS1. On experimental day 2, the mice were subjected to recurrent ASR2. Microdialysates collected at Basal 1 were subjected to metabolite profiling. On experimental day 34, PTSD-like symptoms, including ASR and CF, were assessed.

gesia. Recovery in experimental plexiglass home cages $(16 \times 16 \times$ $32 \mathrm{~cm}^{3}$ ) lasted for 1 week and included Metacam supplementation $(0.25 \mathrm{mg} / 100 \mathrm{ml})$ with drinking water for 3 days after surgery, with daily inspection for food intake. One day before the experiment, the mice were shortly anesthetized with isoflurane. Microdialysis probes with a molecular cutoff of $6 \mathrm{kDa}$ (o.d. $0.2 \mathrm{~mm}$, cuprophane membrane $2 \mathrm{~mm}$ of length, MAB 4.15.2. Cu; Microbiotech/se AB, Stockholm, Sweden) were inserted and connected to the perfusion lines, which consist of FEP tubing and low-volume liquid swivel TCS2-23 (Eicom, Kyoto, Japan). From the moment of insertion, the probes were continuously perfused with sterile artificial cerebrospinal fluid $(\mathrm{NaCl} 145 \mathrm{mM}, \mathrm{KCl} 2.7 \mathrm{mM}$, $\mathrm{CaCl}_{2} 1.2 \mathrm{mM}, \mathrm{MgCl}_{2} 1.0 \mathrm{mM}$ and $\mathrm{Na}_{2} \mathrm{HPO}_{4} 2.0 \mathrm{mM}$; $\left.\mathrm{pH} 7.4\right)$ at a flow rate of $0.3 \mu \mathrm{l} / \mathrm{min}$.

Microdialysis fractions (30 min) were constantly collected at a perfusion rate of $1.5 \mu \mathrm{l} / \mathrm{min}$ into 300 -microliter microtubes (Microbiotech/se AB) following a $1.5 \mathrm{~h}$ equilibration period. During the collection time, the tubes were kept in a refrigerated Univentor 820 Microsampler (Univentor, Zejtun, Malta) at $4^{\circ} \mathrm{C}$. The dead volume of the outlet line was compensated with a delay in fraction sampling $(7 \mathrm{~min})$. The perfusion flow rate was reduced to $0.3 \mu \mathrm{l} /$ min for overnight perfusion.

The microdialysis experiment is outlined in figure 1. On experimental day 1 , the mice were kept in the experimental plexiglass home cages $\left(16 \times 16 \times 32 \mathrm{~cm}^{3}\right)$ for baseline microdialysis sample collection for $1.5 \mathrm{~h}$. The mice were then subjected to a 20 -minute ASR test in a sound and protected environment. To permit a continuous dialysis in microdialysis experiments, we used a custommade startle chamber equipped with a commercially available ASR apparatus (San Diego Instruments, San Diego, Calif., USA). Response was expressed in arbitrary units. At the end of each test, the mice were transferred back to their home cages, and the chamber was cleaned thoroughly with soap water. Two hours later, the mice were subjected to a FS for fear conditioning. Microdialysis was done as described previously [18]. The microdialysate fractions corresponding to the periods of Basal 1 (samples 1-3), ASR1 (samples 4-7) and FS1 (samples 8-11) on experimental day 1 were collected. Only baseline samples were analyzed for metabolite content.

\section{Targeted Metabolomics Analysis}

Four fold (v/v) ice-cold methanol was added to pooled prefrontal microdialysates (samples 1-3 for Basal 1) and vortexed for $2 \mathrm{~min}$. The samples were then incubated for $2 \mathrm{~h}$ on dry ice and centrifuged $\left(2,053 \mathrm{~g}, 10 \mathrm{~min}, 4^{\circ} \mathrm{C}\right)$. Supernatants were transferred to a 0.22 -micrometer cellulose acetate ultrafiltration tube (Corning Life Science, Tewksbury, Mass., USA) and centrifuged $(1,105 g$, $2 \mathrm{~min}, 4^{\circ} \mathrm{C}$ ) to remove undissolved particles. The filtrates were lyophilized and stored at $-80^{\circ} \mathrm{C}$ until further analysis.

The samples were resuspended in $20 \mu \mathrm{LC}$-MS grade water. Ten microliters were injected and analyzed using a 5,500 QTRAP triple quadrupole mass spectrometer (AB/SCIEX, Framingham, Mass., USA) coupled to a Prominence UFLC HPLC system (Shimadzu, Columbia, Md., USA) via SRM of a total of 275 endogenous water soluble metabolites for steady-state analyses of the samples. The samples were delivered to the mass spectrometer via normal-phase chromatography using a $4.6 \mathrm{~mm}$ i.d $\times 10 \mathrm{~cm}$ Amide Xbridge HILIC column (Waters Corp., Milford, Mass., USA) at $350 \mu \mathrm{l} / \mathrm{min}$. Gradients were run starting from $85 \%$ buffer B (HPLC grade acetonitrile) to $42 \% \mathrm{~B}$ from 0 to $5 \mathrm{~min} ; 42$ to $0 \% \mathrm{~B}$ from 5 to $16 \mathrm{~min} ; 0 \% \mathrm{~B}$ was held from 16 to $24 \mathrm{~min} ; 0$ to $85 \% \mathrm{~B}$ from 24 to $25 \mathrm{~min} ; 85 \% \mathrm{~B}$ was held for $7 \mathrm{~min}$ to re-equilibrate the column. Buffer A was comprised of $20 \mathrm{mM}$ ammonium hydroxide $/ 20 \mathrm{~mm}$ ammonium acetate, pH 9.0, in 95:5 water:acetonitrile. Some metabolites were targeted in both positive and negative ion modes for a total of 292 SRM transitions using positive/negative polarity switching. ESI voltage was $+4,900 \mathrm{~V}$ in the positive ion mode and $-4,500 \mathrm{~V}$ in the negative ion mode. The dwell time was $4 \mathrm{~ms}$ per SRM transition, and the total cycle time was $1.89 \mathrm{~s}$. Approximately 9-12 data points were acquired per detected metabolite. Peak areas from the total ion current for each metabolite SRM transition were integrated using MultiQuant v2.0 software (AB/Sciex).

\section{Statistics and Data Analysis}

Metabolites with $>20 \%$ missing value and $z$-score $>4$ were considered outliers and removed from our analyses. We normalized the intensities of each metabolite to have a mean of zero and a standard deviation of 1 . Correlation analyses for the metabolite levels and behavioral readouts were performed employing GraphPad Prism 5 (GraphPad Software, La Jolla, Calif., USA). In brief, we correlated Basal 1 metabolite levels with behavioral readouts (ASR2/ASR1, ASR34 and CF34) by calculating the Pearson correlation coefficient. The significant level was set to $p<0.05$. Pathway analysis was conducted using MetaboAnalyst pathway tool (http:// www.metaboanalyst.ca).

\section{Results}

\section{Metabolite Profiling of mPFC Extracellular Fluid}

Microdialysate fractions were subjected to highthroughput metabolomics analyses with the help of a targeted LC-MS platform interrogating a list of close to 300 metabolites that encompass several central pathways related to energy metabolism, including citrate cycle, glycolysis or gluconeogenesis and pyruvate metabolism. In addition, the list included a number of central nervous 


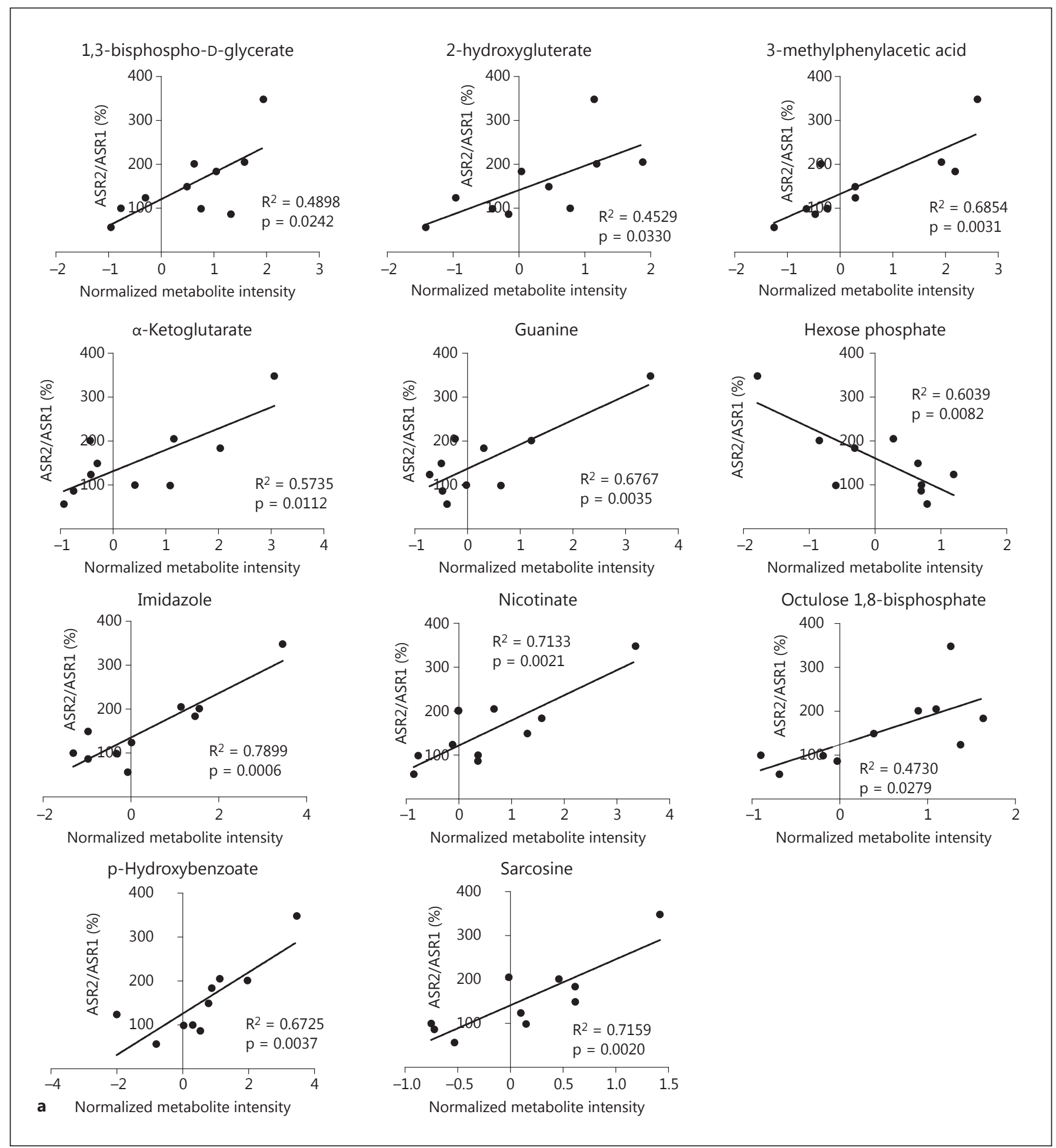

Fig. 2. Linear correlations between Basal 1 metabolite levels and FS-induced behavioral changes. Basal 1 metabolite levels correlated with FS-induced ASR2/ASR1 (a), ASR34 (b) and CF34 (c).

(For Figure $2 b$ and $c$ see next page.)

Applying Modern Metabolomics to the Analysis of Extracellular Fluid
Mol Neuropsychiatry 2015;1:60-67 DOI: $10.1159 / 000381855$ 

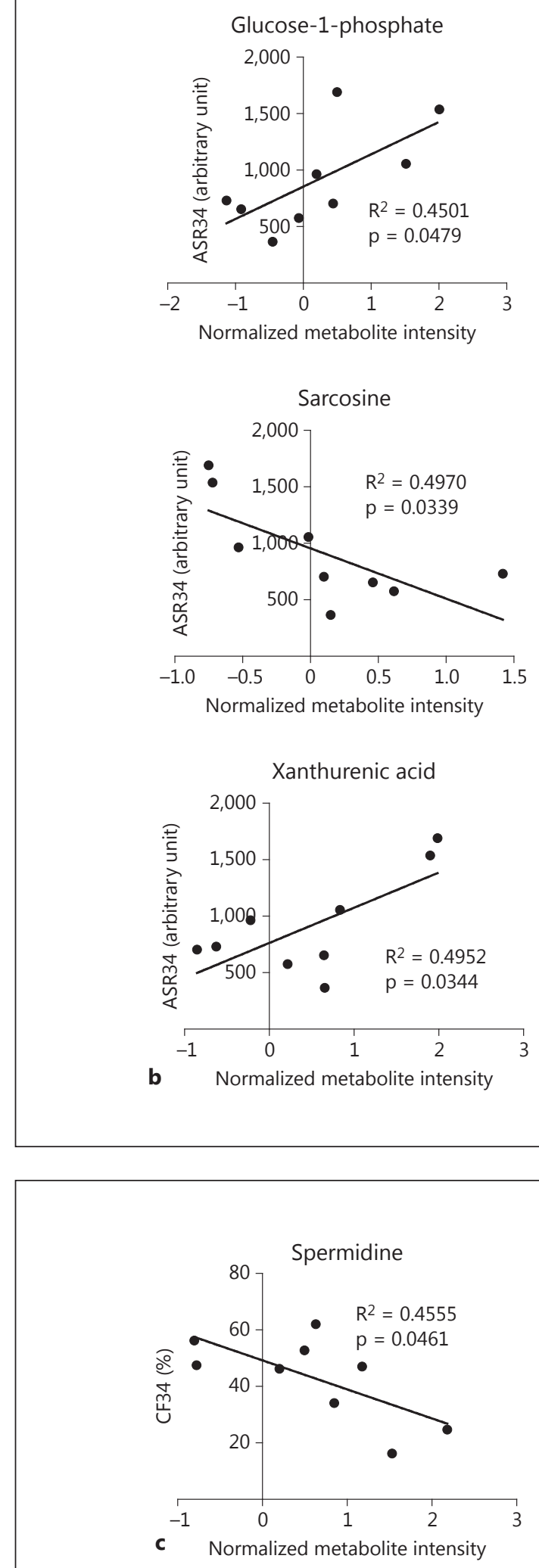

2

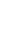

Table 1. Pathways enriched by metabolites correlating with FSinduced ASR2/ASR1 and metabolites with a correlating expression profile

\begin{tabular}{lll}
\hline Pathway & $\begin{array}{l}\text { Holm-Bonferroni- } \\
\text { adjusted p value }\end{array}$ & $\begin{array}{l}\text { False } \\
\text { discovery } \\
\text { rate }\end{array}$ \\
\hline $\begin{array}{l}\text { Citrate cycle } \\
\begin{array}{l}\text { Glyoxylate and dicarboxylate } \\
\text { metabolism }\end{array}\end{array}$ & 0.0002 & 0.0002 \\
\hline
\end{tabular}

system-relevant molecules that are typically analyzed in microdialysis experiments including neurotransmitters (glutamate and glycine) and endogenous ion channel agonists/antagonists (aspartate, alanine, kynurenic acid and phenylalanine) (online suppl. table S1; see wwww. karger.com/doi/10.1159/000381855 for all online supplementary material). In our PTSD mouse model, we were able to quantify 120 metabolites after data filtering and normalization (online suppl. table S1).

mPFC Metabolic Fingerprints under Basal Conditions Predict FS Impact on Arousal

Metabolic profiling of prefrontal extracellular fluid allowed us to correlate molecular data with behavioral measures in the PTSD mouse model. We identified several central metabolites, which showed linear correlations with the FS-related early changes in arousal (ASR2/ASR1; fig. 2a). We then subjected the metabolites that predicted the FS-induced arousal changes and the highly correlating expression profile metabolites (online suppl. table S2) to pathway analyses. Citrate cycle metabolism and glyoxylate and dicarboxylate metabolism were significantly enriched (table 1). These findings demonstrate the power of metabolite profiling for predicting early behavioral consequences of FS and investigating affected pathways in the PTSD mouse model.

mPFC Metabolic Fingerprints under Basal Conditions Predict Long-Term PTSD-Like Symptoms

In addition to the identified correlations for FS-induced early changes in arousal, a number of central metabolites showed prediction power for longer-term PTSD-symptoms, including ASR and CF 34 days after FS (fig. 2b, c). Basal 1 xanthurenic acid and glucose-1-phosphate levels positively predicted ASR34, while sarcosine levels negatively predicted ASR34. Basal 1 spermidine levels negatively predicted the CF34 outcome. 


\section{Discussion}

The analysis of brain microdialysate samples from individual mice with an LC-MS metabolomics platform enabled us to detect and quantify the levels of close to 120 metabolites. Our approach significantly extends the spectrum of microdialysate analytes and is able to delineate molecular pathways involved in pathological changes related to brain metabolism in a mouse model for PTSD. Metabolomics data analysis revealed numerous correlations between basal mPFC metabolite profiles and FS-associated arousal and contextual fear. Our results demonstrate that combining microdialysis with LC-MS provides a sensitive approach of a high-prediction power for deciphering the molecular signatures associated with individual susceptibility for developing PTSD-like symptoms in a given brain region. In an extension of this approach, microdialysis experiments could provide important insights into affected brain circuits underlying pathophysiological mechanisms by targeting different brain regions.

Microdialysis has been widely applied for the evaluation of extracellular dynamics of neurotransmitters and pharmacological substances in defined brain regions. Targeted quantification of up to 20 monoamines and their metabolites from microdialysis is feasible but provides rather limited insights for specific biological questions [18]. Microdialysis is also routinely used to explore changes in the extracellular levels of neuroactive amino acids including aspartate, glutamate, glutamine and alanine that are relevant not only for neurotransmission focused studies but also for neurotoxicity experiments [19, 20]. However, for sensitivity reasons, the electrochemical or fluorescent detection of amino acids in microdialysates requires pre- or post-column derivatization, which can lead to sample loss and/or measurement variability. In the present study, we were able to quantitate all 20 natural and several additional modified amino acids without derivatization by applying modern metabolomics LC-MS technology as readout for the comprehensive profiling of brain microdialysates (online suppl. table S1). This allowed us to reveal significant amino acid pathways affected by stressors and associated with PTSD-like symptom severity (table 1 ).

In an earlier study [21] applying the same experimental design, no significant linear correlations were found between basal prefrontal norepinephrine and corticosterone levels and behavioral responses to stress. When analyzing the here presented metabolomics data, Pearson correlation analysis showed prediction power for the metabolites with regard to behavioral changes of the PTSD

Applying Modern Metabolomics to the

Analysis of Extracellular Fluid mice (fig. 2). The correlations between baseline metabolite levels and FS-induced arousal and PTSD-like symptoms demonstrate the power of microdialysate metabolite profiling for the investigation of interindividual variability, in this case susceptibility or resilience towards coping with psychophysiological stress and the development of PTSD endophenotype, in an inbred mouse strain.

Several of the metabolites that show correlations to the early (day 2) and delayed (day 34) behavioral changes induced by stress have relevance for psychiatric phenotypes. Sarcosine, a glycine reuptake inhibitor, has been implicated in the regulation of synaptic plasticity, memory and cognition [22]. Our data show a positive correlation between Basal 1 sarcosine levels and FS-induced ASR2/ASR1. On the other hand, Basal 1 sarcosine levels negatively predicted the FS-induced ASR34. These data suggest that sarcosine-dependent prefrontal mechanisms of plasticity show biphasic dynamics with respect to arousal state regulation. We were also able to quantify neurokynurenines, including kynurenic acid, xanthurenic acid and nicotinate from prefrontal microdialysates. The kynurenine pathway is the major route for tryptophan metabolism and has been implicated in the pathophysiology of depression and stress-related disorders [23, 24]. Mice subjected to unpredictable chronic mild stress exhibited an elevation of the peripheral kynurenine pathway [25]. In the same study, a correlation between the peripheral kynurenine/tryptophan ratio and anxiodepressive-like behavior in mice subjected to unpredictable chronic mild stress was found. Previous studies showed that treatment with nicotinate resulted in improved human sensory register and short-term memory, possibly by facilitating neurotransmission [26]. By binding with neuronal transporters, nicotinate can be utilized as cellular precursor for nicotinamide adenine nucleotide and nicotinamide adenine dinucleotide phosphate synthesis, key events in regulating energy metabolism that has been implicated in the pathogenesis of psychiatric disorders [27-29]. In the current study, we found prefrontal nicotinate levels to positively correlate with FS-induced ASR2/ ASR1 change and xanthurenic acid levels to positively predict the arousal response 34 days after shock (ASR34). These results support a possible role for neurokynurenines in mediating neuronal activity, specifically relevant for arousal mechanisms.

An additional benefit of using a great number of analytes for the interrogation of microdialysates is the ability to get information about affected molecular pathways relevant for symptom development in the mouse model under investigation. When subjecting the enriched metabo- 
lites to pathway analyses, we found energy metabolism, specifically the citrate cycle pathway, to be affected in the PTSD mouse model. Previous studies have shown increased citrate cycle activity in synaptosomes of highanxiety mice [30]. Functional synapses are enriched with mitochondria, which supply ATP and regulate calcium levels, modulating neuronal transmembrane potential [31], synaptic neurotransmission [32], receptor signaling [33] and synaptic plasticity [34]. A dysregulated citrate cycle metabolism leads to abnormal energy production that can impair neurotransmission. This may be of particular relevance in the $\mathrm{mPFC}$, which exerts top-down control of emotional states. Anxiolytic medications such as monoamine oxidase inhibitors were shown to improve mitochondrial function [35]. Based on our findings, we assume that differential regulation of the citrate cycle may be involved in endophenotype-specific neurotransmission in the PTSD mouse model.

With the help of the LC-MS metabolomics platform, we were also able to quantify a number of metabolites that are directly related to neurotransmission, including glutamate, hydroxyphenylacetic acid, phenylalanine, trypto- phan and the N-methyl-D-aspartate receptor agonists alanine and aspartate. Further applications of the LC-MS metabolomics platform could include studies of neurotransmitter dynamics and help delineate affected pathways involved in neuronal plasticity.

LC-MS microdialysate analysis yields high sensitivity and selectivity compared to the commonly used detection methods [36]. The method allows a reliable qualitative and quantitative determination of a wide range of metabolites. With the help of the LC-MS metabolomics platform, we were able to reveal alterations in intracellular metabolic pathways reflected as changes in metabolite profiles in the extracellular fluid. The method enables hypothesis-free physiological studies, is applicable to any rodent model and may illuminate novel targets for pharmacological intervention.

\section{Acknowledgements}

This work was supported by the Max Planck Society. C.-Y.K. was supported by the LMU Graduate School of Systemic Neurosciences.

\section{References}

$>1$ Rittenhouse KD, Pollack GM: Microdialysis and drug delivery to the eye. Adv Drug Deliv Rev 2008;45:229-241.

-2 Tsai TH, Cheng FC, Chen YF, Chen CF: Online microdialysis coupled with microbore liquid chromatography with ultraviolet detection for continuous monitoring of free cefsulodin in rat blood. J Chromatogr A 2001;914: 83-87.

$>3$ Chang YL, Chou MH, Lin MF, Chen CF, Tsai $\mathrm{TH}$ : Determination and pharmacokinetic study of meropenem in rat bile using on-line microdialysis and liquid chromatography. J Chromatogr A 2002;961:119-124.

4 Ungerstedt U, Pycock C: Functional correlates of dopamine neurotransmission. Bull Schweiz Akad Med Wiss 1974;30:44-55.

$\checkmark 5$ Anderzhanova E, Wotjak CT: Brain microdialysis and its applications in experimental neurochemistry. Cell Tissue Res 2013;1:27-39.

-6 Westerhout J, Ploeger B, Smeets J, Danhof M, de Lange EC: Physiologically based pharmacokinetic modeling to investigate regional brain distribution kinetics in rats. AAPS J 2012;14:543-553.

$>7$ Guihen E, O'Connor WT: Current separation and detection methods in microdialysis the drive towards sensitivity and speed. Electrophoresis 2009;30:2062-2075.

$>8$ Zhang MY, Hughes ZA, Kerns EH, Lin Q, Beyer CE: Development of a liquid chroma- tography/tandem mass spectrometry method for the quantitation of acetylcholine and related neurotransmitters in brain microdialysis samples. J Pharm Biomed Anal 2007;44: 586-593.

$>9$ Hows ME, Lacroix L, Heidbreder C, Organ AJ, Shah AJ: High-performance liquid chromatography/tandem mass spectrometric assay for the simultaneous measurement of dopamine, norepinephrine, 5-hydroxytryptamine and cocaine in biological samples. J Neurosci Methods 2004;138:123-132.

10 Berna MJ, Ackermann BL: Quantification of serine enantiomers in rat brain microdialysate using Marfey's reagent and LC/MS/MS. J Chromatogr B 2007;846:359-363.

11 Haskins WE, Wang Z, Watson CJ, Rostand RR, Witowski SR, Powell DH, Kennedy RT: Capillary LC-MS2 at the attomole level for monitoring and discovering endogenous peptides in microdialysis samples collected in vivo. Anal Chem 2001;73:5005-5014.

12 Dias C, Nashed Y, Atluri H, Mitra A: Ocular penetration of acyclovir and its peptide prodrugs valacyclovir and val-valacyclovir following systemic administration in rabbits: an evaluation using ocular microdialysis and LCMS. Curr Eye Res 2002;25:243-252.

$>13$ Chen YF, Chang CH, Wang SC, Tsai TH: Measurement of unbound cocaine in blood, brain and bile of anesthetized rats using mi- crodialysis coupled with liquid chromatography and verified by tandem mass spectrometry. Biomed Chromatogr 2005;19:402-408.

14 Bengtsson J, Jansson B, Hammarlund-Udenaes $\mathrm{M}$ : On-line desalting and determination of morphine, morphine-3-glucuronide and morphine-6-glucuronide in microdialysis and plasma samples using column switching and liquid chromatography/tandem mass spectrometry. Rapid Commun Mass Spectrom 2005; 19:2116-2122.

$>15$ Siegmund A, Wotjak CT: A mouse model of posttraumatic stress disorder that distinguishes between conditioned and sensitized fear. J Psychiatr Res 2007;41:848-860.

-16 Golub Y, Mauch CP, Dahlhoff M, Wotjak CT: Consequences of extinction training on associative and non-associative fear in a mouse model of posttraumatic stress disorder (PTSD). Behav Brain Res 2009;205:544549.

17 Paxinos G, Franklin KBJ: A Stereotaxic Atlas of the Mouse Brain. San Diego, Academic Press, 2002.

18 Anderzhanova E, Bächli H, Buneeva OA, Narkevich VB, Medvedev AE, Thoeringer CK, Wotjak CT, Kudrin VS: Strain differences in profiles of dopaminergic neurotransmission in the prefrontal cortex of the BALB/C vs. $\mathrm{C} 57 \mathrm{Bl} / 6$ mice: consequences of stress and afobazole. Eur J Pharmacol 2013;708:95-104. 
19 Wölfer J, Gorji A, Speckmann EJ, Wassmann $\mathrm{H}$ : Interstitial amino acid concentrations in rodent brain tissue during chemical ischemia. J Neurosci Res 2014;92:955-963.

20 Anderzhanova E, Rayevsky KS, Saransaari P, Riitamaa E, Oja SS: Effects of acute toxic doses of psychostimulants on extracellular levels of excitatory amino acids and taurine in rats: comparison of d-amphetamine and sydnocarb. Ann NY Acad Sci 2002;965:193-203.

21 Kao C-Y, Stalla G, Stalla J, Wotjak CT, Anderzhanova E: Norepinephrine and corticosterone in the medial prefrontal cortex and hippocampus predict PTSD-like symptoms in mice. Eur J Neurosci 2015, E-pub ahead of print.

22 McDonald JW, Johnston MV: Physiological and pathophysiological roles of excitatory amino acids during central nervous system development. Brain Res Rev 1990;15:41-70.

23 Curzon G, Bridges PK: Tryptophan metabolism in depression. J Neurol Neurosurg Psychiatry 1970;33:698-704.

24 Lapin IP: Neurokynurenines (NEKY) as common neurochemical links of stress and anxiety. Adv Exp Med Biol 2003;527:121-125.

25 Laugeray A, Launay JM, Callebert J, et al: Evidence for a key role of the peripheral kynurenine pathway in the modulation of anxiety- and depression-like behaviours in mice: focus on individual differences. Pharmacol Biochem Behav 2011;98:161-168.
26 Loriaux SM, Deijen JB, Orlebeke JF, De Swart JH: The effects of nicotinic acid and xanthinol nicotinate on human memory in different categories of age. A double blind study. Psychopharmacology (Berl) 1985;87:390-395.

27 Mallei A, Failler M, Corna S, Racagni G, Mathé AA, Popoli M: Synaptoproteomic analysis of a rat gene-environment model of depression reveals involvement of energy metabolism and cellular remodeling pathways. Int J Neuropsychopharmacol 2014; 18:pyu067.

28 Einat H, Yuan P, Manji HK: Increased anxiety-like behaviors and mitochondrial dysfunction in mice with targeted mutation of the Bcl-2 gene: further support for the involvement of mitochondrial function in anxiety disorders. Behav Brain Res 2005; 165:172-180.

29 Mancuso C, Scapagini G, Currò D, Giuffrida Stella AM, De Marco C, Butterfield DA, Calabrese V: Mitochondrial dysfunction, free radical generation and cellular stress response in neurodegenerative disorders. Front Biosci 2007;12:1107-1123.

30 Filiou MD, Zhang Y, Teplytska L, Reckow S, Gormanns P, Maccarrone G, Frank E, Kessler MS, Hambsch B, Nussbaumer M, Bunck M, Ludwig T, Yassouridis A, Holsboer F, Landgraf R, Turck CW: Proteomics and metabolomics analysis of a trait anxiety mouse model reveals divergent mitochondrial pathways. Biol Psychiatry 2011;70:1074-1082.
31 Mattson MP, Partin J: Evidence for mitochondrial control of neuronal polarity. J Neurosci Res 1999;56:8-20.

32 Billups B, Forsythe ID: Presynaptic mitochondrial calcium sequestration influences transmission at mammalian central synapses. J Neurosci 2002;22:5840-5847.

33 Kann O, Kovacs R, Heinemann U: Metabotropic receptor-mediated $\mathrm{Ca} 2+$ signaling elevates mitochondrial $\mathrm{Ca} 2+$ and stimulates oxidative metabolism in hippocampal slice cultures. J Neurophysiol 2003;90:613-621.

34 Vanden Berghe P, Kenyon JL, Smith TK: Mitochondrial $\mathrm{Ca} 2+$ uptake regulates the excitability of myenteric neurons. J Neurosci 2002; 22:6962-6971.

35 Thiffault C, Quirion R, Poirier J: The effect of L-deprenyl, D-deprenyl and MDL72974 on mitochondrial respiration: a possible mechanism leading to an adaptive increase in superoxide dismutase activity. Brain Res Mol Brain Res 1997;49:127-136.

36 Hows ME, Organ AJ, Murray S, Dawson LA, Foxton R, Heidbreder C, Hughes ZA, Lacroix L, Shah AJ: High-performance liquid chromatography/tandem mass spectrometry assay for the rapid high sensitivity measurement of basal acetylcholine from microdialysates. J Neurosci Methods 2002;121:33-39.
Applying Modern Metabolomics to the Analysis of Extracellular Fluid
Mol Neuropsychiatry 2015;1:60-67 